\title{
DYNAMO IN ASTROPHYSICS
}

\author{
A.A.RUZMAIKIN \\ IZMIRAN, Academy of Sciences \\ Troitsk \\ Moscow Region, 142092 \\ USSR
}

\begin{abstract}
The fast dynamo acting in a turbulent flow explains the origin of magnetic fields in astrophysical objects. Stellar cycles and large-scale magnetic fields in spiral galaxies reflect the behaviour of a mean magnetic field. Intermittent magnetic structures in clusters of galaxies are associated with random magnetic field.
\end{abstract}

\section{THE CONCEPT OF THE FAST DYNAMO}

Astrophysics differs from the laboratory physics first of all by the astronomical scale. It creates new qualities. One of them, a triumph of plasma hydrodynamical motions over the molecular dissipation can explain the origin of magnetic fields in many astrophysical bodies like stars and galaxies.

The inductive action of motions has a characteristic time $\ell / v$, where $\ell$ and $v$ are the scale and amplitude of the velocity field. A characteristic dissipation time is $\ell^{2} / \nu_{m}$, where $\nu_{m}$ is a magnetic diffusivity. The dimensionless ratio of the times, $\ell v / \nu_{m}$, called the magnetic Reynolds number $R_{m}$, is very large for astrophysical conditions. For example, in the solar convective shell it is $10^{8}$. The magnetic field appears to be practically frozen in the flow.

Frozenness of the magnetic field prevents a creation of new magnetic lines needed for the field amplification in a regular flow, say, when the streamlines lie on stationary surfaces. A finite $R_{m}$ gives the possibility to create magnetic lines however a rate of the magnetic field growth is very small at large $R_{m}$ (the slow dynamo, Zeldovich and Ruzmaikin, 1980).

Fortunately, astrophysical flows are highly turbulent because the Reynolds number at the same scale is also very large. In the highly conducting flows the rate of magnetic field growth tends to a finite value when $R_{m}$ goes to infinity. It may seem paradoxical but the complexity of the flow simplifies the problem of magnetic field generation. The idea is similar to that used in the central limit theorem of the probability theory where a large number of random quantities results in appearance of a regular mean arithmetic value and the normal distribution for deviations from this value. In fact, random flow has a characteristic 
correlation time $\tau$ (a memory time). During this time the random flow acts on an initial magnetic field at a given fluid particle rather systematically turning the field by some angle and amplifying or weakeaning its intensity. The resulting magnetic field can be considered as initial one for the next independent step with the same duration $\tau$ and so on. After a large number of such steps one will obtain a product of independent random operators acting on the initial magnetic field. The products can acquire the very large values at some places of sufficiently big volume. Moreover, it can be shown for some natural conditions imposed on the velocity field that almost every product will grow when the number of steps, i.e. the time, increases. However, each realization has its own rate of growth so that the resulting magnetic structure becomes intermittent (Molchanov et al., 1984).

By visual demonstration one may imagine a set of flux tubes distributed in the large volume. At some places the tubes expand, take the eight form, and fold to double the field strength and the magnetic flux through their cross-sections (Zeldovich's eight dynamo). At many other places the parts of tubes with opposite field directions meet each other giving rise to reconnections which destroy the tubes and diminish the magnetic energy (Figure 1). All it takes place in a "well mixed" isotropic random flow.

When the velocity field has a non zero correlation 〈v rotv〉 called a magnetic helicity, the averaged, mean magnetic field can be excited. The

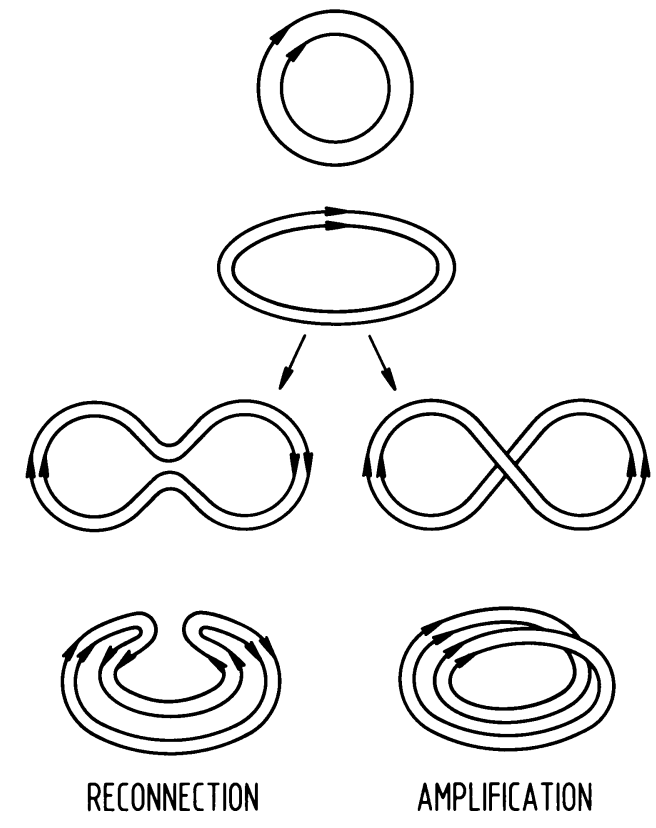

Figure 1. A simple demonstration of the magnetic loop transformations by random flow. 
regular components of the velocity field, for example a differential rotation, also contribute to generation of this field.

Analysis of the mean magnetic field behaviour is very similar conceptually to thermodynamics: everything from the turbulence is hidden in the coefficients like turbulent viscosity and mean helicity (Moffatt, 1978; Parker, 1979; Krause and Rädler, 1980). However, this "magnetic thermodynamics" can give suprising results, for example the strange attractor behaviour for the mean solar magnetic field which manifests itself in the form of the Grand Minima in the solar activity (Ruzmaikin, 1981; Zeldovich et al., 1983; Weiss et al., 1984).

In this short review only some aspects of the astrophysical dynamos are considered in particular the role of the mean magnetic field in the stellar activity cycles and the large-scale magnetic structures in spiral galaxies. The "small-scale", intermittent magnetic fields are discussed in application to the clusters of galaxies (Sokoloff et al., this volume).

\section{STELLAR ACTIVITY CYCLES}

One old astromoner once told me that first and foremost of astronomy are the stars. Paying tribute to this point of view it is natural to start with a role of magnetic field in stars.

Observations show that magnetic fields provide activity in stars and also in the area around them. For a long time the solar activity cycle was the only observed magnetic cycle. Measurements of $H$ and $K$ lines of singly ionized calcium in a variety of late-type stars reveal cyclic variations of the emission with the periods 7-12 years (Wilson, 1978; Baliunas and Vaughan, 1985).

The mean magnetic field is self-excited in a stellar convective, turbulent shell due to two sources: the mean helicity and differential rotation. In spite of lack of a developed theory of turbulent convection it is possible to make some crude estimates of distributions of the sources.

According to the mixing-length theory of the stellar convective zones (Baker and Tamesvary, 1966) distribution of the turbulent diffusivity, $v_{t}$, is almost uniform. As shown by modern helioseismology the gradients of the solar angular velocity, $\Omega$, are pronounced near the bottom of the convective shell.

The mean helicity arises due to the action of rotation (through the Coriolis force) on convecting stratified fluid. When the lifetime of the main vortices, $\ell / v$, is shorter than the period of the rotation, $\Omega^{-1}$, i.e. the Rossby number is large, the correlator $\alpha=-\tau\langle v$ rotv $\rangle$ is approximately equal to $\ell \Omega$. In the opposite case, for a small Rossby number, an averaging over the rotation period gives $\alpha=v$. The expected distribution of the mean helicity along the depth of the convective zone is shown in Figure 2. It is a very good hydrodynamical problem to calculate this distribution more professionally.

After taking into account the condition $v_{\mathrm{t}}=\ell \mathrm{v}=$ constant one obtains $\alpha_{\max } \propto v\left(r_{\max }\right) \propto \Omega^{1 / 2}$. Another important conclusion from Figure 2 is: the mean helicity maximum is shifted to the star surface for slowly rotating stars, and it is near the bottom of the convective zone for the 


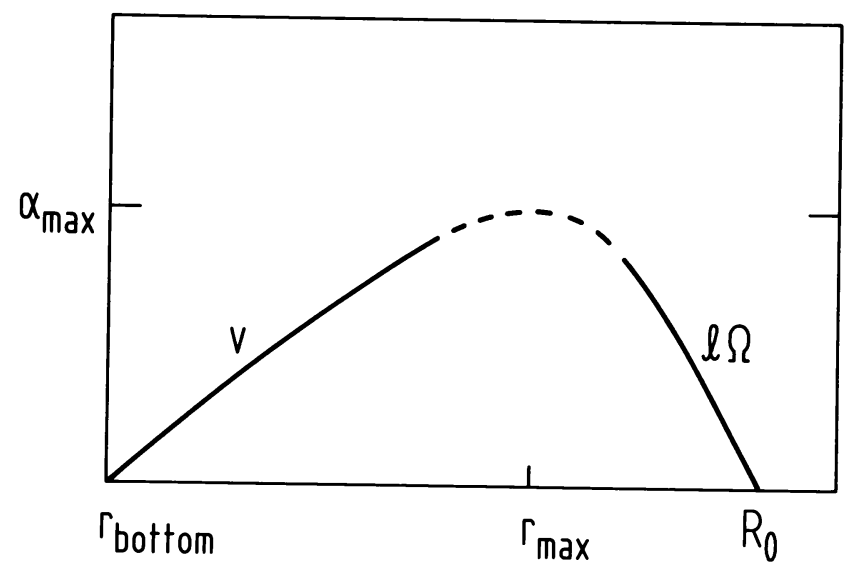

Figure 2. The schematic diagram of the mean helicity distribution in stellar convective shell. The mean helicity reaches its maximum at a radius $r_{\max }$ where $v$ is of the order of $\ell \Omega$.

fast rotating stars. Thus, in the case of slow rotators the mean magnetic field is generated by spatially separated sources $(\nabla \Omega$ and $\alpha)$, for the fast rotating convective zones the field is excited by overlapping sources.

The intensity of the sources is characterized by the dimensionless dynamo number:

$$
\mathrm{D}=\alpha \nabla \Omega \mathrm{R}^{4} \quad v_{\mathrm{t}}^{-2} \infty \Omega^{3 / 2}
$$

Solutions of the mean dynamo equations have the form of magnetic waves. The period of the oscillations, which can be identified with the observed stellar cycle period, differently depends on the dynamo number in two above mentioned cases: $T \propto D^{-2 / 3} \propto \Omega^{-1}$ for the slowly rotating stars, and $T \propto \ln ^{-1} \mathrm{D}$ (i.e. practically independent of the angular velocity) in the case of the fastly rotating stars of the same spectral type (Kleeorin et al., 1983; Kleeorin and Ruzmaikin, 1988). This result can be directly confronted with observations. Dynamo models for the stars of different spectral types are considered by Belvedere et al. (1980).

Other manifestations of stellar activity are hot coronae observed in $\mathrm{X}$-rays. The most probable mechanism for the corona heating is associated with reconnections in magnetic loops detached from the magnetic field generated in the star convective zone. Efficiency of the energy release is proportional to $10^{-2} \mathrm{~B}^{2} / 8 \pi\left(\mathrm{v}_{\mathrm{A}} / \mathrm{L}_{1 \text { oop }}\right)$ (Galeev et al., 1981). According to a simple non-linear dynamo model, the magnetic field amplitude is proportional to $\mathrm{D}^{1 / 2}$. Combining this result with the scaling law (1) one obtains

$$
L_{X} \propto B^{3} \propto \Omega^{9 / 4}
$$


(Kleeorin and Ruzmaikin, 1988). It is close to a $\Omega^{2}$ dependence $(9 / 4=$ $2+1 / 4)$ as pointed out by Pallavichini et al. (1981).

\section{GEOMETRY OF THE GALACTIC DYNAMO}

The only dynamo region we are living within is the Galactic one. It gives a unique possibility for observers to study the velocity and magnetic field distributions in the dynamo region and leaves no room for ambiguous theoretical speculations.

Distributions of the gas angular velocity along the disk radius in spiral galaxies, the rotation curves, are known from the $21 \mathrm{~cm}$ radio observations. However, the distributions of the mean helicity are yet a matter of theoretical speculations (Ruzmaikin et al., 1988).

It is a very important but not simple problem to find a way to determine the mean helicity directly from the observed velocity field.

The shape of the galactic disk plays an important role in galactic dynamos. In our Galaxy, for example, the disk cannot be considered as a limiting case of an ellipsoid as soon as its meridional cross-section has a hyperboloidal form, i.e. the thickness of the disk increases outside (Figure 3). The dynamo models for an axisymmetric disk was studied in detail (Ruzmaikin et al., 1988).

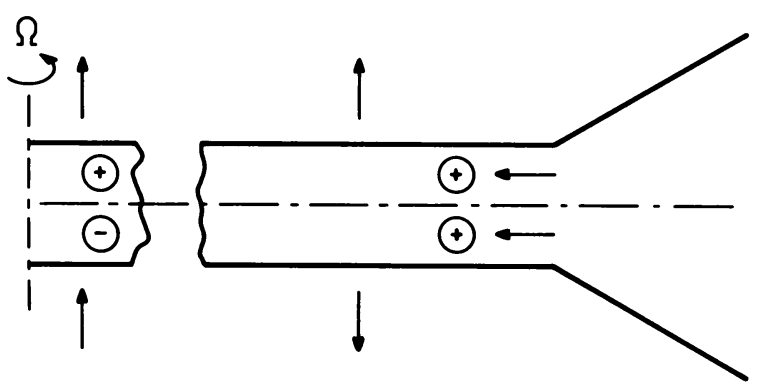

Figure 3. The shape of the gas disk in the Galaxy. The arrows show directions for $B_{r}, B_{z}$ and circles for $B_{\downarrow}$ components of the mean magnetic field.

Observational discovery of global magnetic structures in a number of spiral galaxies (see reviews Sofue et al., 1986; Beck, 1986; and $M$. Krause, this volume) is an essential step in development of modern astrophysics of galaxies. The main modes suggested by the observations are the axisymmetric, $m=0$, and bisymmetric, $m=1$, ones. The dynamo in an axisymmetric differentially rotating gas disk excites, as the first preferable mode, the axisymmetric magnetic field. There are two galaxies, M31 and IC 342, where the observed magnetic configurations are the axisymmetric ones. However, the structures in M81 and in M51 are the bisymmetric modes ( $M$. Krause, this volume). These results are a challenge 
to theoreticians. It is necessary to leave the full axisymmetry of the disk. Among the ways to do it there are: to take into account the spiral structure, the tidal disturbances of the disk, etc.

An interesting observational problem is to determine a distribution of $\mathrm{B}_{z}$ component along the disk. The theory predicts the even symmetry (the same signs over and under the disk) in the thin disk approximation. In the central part of the galaxy this approximation does not work, and in the thick disk here a dynamo probably will excite the odd mode of the poloidal magnetic field as preferable. The observations evidence in favor of the odd poloidal field in the central part of the Galaxy (Sofue et al., Reich, this volume).

The other interesting problem both from the observational and theoretical points of views is a study of magnetic fields in dwarf galaxies in which the rotation almost uniform and a ratio of the tubulent and rotational velocities is much larger than in spirals (U. Klein, private communication). LMC having many attractive features ( $R$. Wielebinski, private discussion) is a separate headache for the dynamo experts.

\section{INTERMITTENT MAGNETIC FIELDS IN CLUSTERS OF GALAXIES}

The non-thermal radio emission from several clusters of galaxies evidence on the presence of magnetic fields in them. The origin of these fields is discussed in detail in the recent paper by Ruzmaikin, Shukurov and Sokoloff (1989), see also Sokoloff et al. (this volume).

The gas motions needed to generate the magnetic fields appear in the turbulent wakes behind the galaxies moving through the cluster. Overlapping wakes give a picture of well developed turbulence of the Kolmogorov type. For example, in the Coma cluster 150 galaxies moving with a speed of about $10^{3} \mathrm{~km} / \mathrm{s}$ and having the initial wake radius $10 \mathrm{kpc}$ can fill with this turbulence a volume of $300 \mathrm{kpc}$ in size. A turbulent velocity at the energy-range scale is of about $400 \mathrm{~km} / \mathrm{s}$. In the Cygnus A cluster a number of galaxies observed is much less compared to the Coma cluster. A careful analysis is needed to explain the origin of turbulence here.

There is no mean helicity in the clusters of galaxies so that the generation of the mean magnetic field is not possible. Instead the turbulence generates the spatially and temporary intermittent magnetic fields (see Section 1 and Figure 1). The field distribution can be considered as relatively strong concentrated ropes of a skin-layer thickness $\ell R_{m}-1 / 2$, where $\ell$ is a characteristic scale of the turbulence, with a weak background field in between. A strength of the field in the ropes can be estimated from the condition of equipartition betweeen the magnetic and kinetic energy densities, $\mathrm{H}_{\mathrm{eq}} \approx 2 \mu \mathrm{G}$. A contribution of the intermittent magnetic field to the line of sight Faraday rotation measure is, of course, very small, $\left\langle\mathrm{H}^{2}\right\rangle^{1 / 2} \approx 0.2 \mu \mathrm{G}$.

The dynamo process to generate the intermittent magnetic fields is surely acting in the high magnetic Reynolds plasmas in the convective shells of stars (it may explain the origin of the starspots), in the galaxies (to create small scale structures in the galactic magnetic field) and in the astrophysical jets. 


\section{REFERENCES}

Baker, N. and Tamesvary, S. (1966) Tables of stellar envelope models, New York.

Baliunas, S.L. and Vaughan, A.H. (1985) Ann. Rev. Astron. Astrophys. 23, 379-412.

Beck, R. (1986) IEEE Trans. Plasma Sci. 14, 740-747.

Belvedere, G., Paterno, L. and Stix, M. (1980) Astron. Astrophys. 91, 328-330.

Galeev, A.A., Rosner, R. and Vaiana, G.S. (1981) Astrophys. J. 243, 301-308.

Kleeorin, N.I., Ruzmaikin, A.A. and Sokoloff, D.D. (1983) Astrophys. Space Sci. $95,131-136$.

Kleeorin, N.I. and Ruzmaikin, A.A. (1988) in Yu.V. Glagolevsky and I.M. Kopylov (eds.), Magnetic Stars, Nauka, Leningrad, pp. 321-328.

Krause, F. and Rädler, K.-H. (1980) Mean-Field Magnetohydrodynamics and Dynamo Theory, Pergamon Press, Oxford.

Moffatt, H.K. (1978) Magnetic Field Generation in Electrically Conducting Fluids, Cambridge Univ. Press, Cambridge.

Molchanov, S.A., Ruzmaikin, A.A. and Sokoloff, D.D. (1983) Geophys. Astrophys. Fluid Dyn. 30, 241-252.

Pallavichini, R., Golub, L., Rosner, R., Vaiana, G.S., Ayres, T. and Linsky, J.L. (1981) Astrophys. J. 248, 279-290.

Parker, E.N. (1979) Cosmic Magnetic Fields, Oxford Univ. Press, Oxford.

Ruzmaikin, A.A. (1981) Comments on Astrophys. 9, 85-91.

Ruzmaikin, A.A., Shukurov, A.M. and Sokoloff, D.D. (1988) Magnetic Fields of Galaxies, Kluwer Academic Publishers, Dordrecht.

Ruzmaikin, A.A., Shukurov, A.M. and Sokoloff, D.D. (1989) Monthly Notices Roy. Astron. Soc. (in press).

Sofue, Y., Fujimoto, M. and Wielebinski, R. (1986) Ann. Rev. Astron. Astrophys. 24, 459-498.

Weiss, N.O., Cattaneo, F. and Jones, C.A. (1984) Geophys. Astrophys. Fluid Dyn. 30, 305.

Wilson, O. (1978) Astrophys. J. 226, 379-396.

Zeldovich, Ya.B. and Ruzmaikin, A.A. (1980) Sov. Phys. JETP 51, 493-497.

Zeldovich, Ya.B., Ruzmaikin, A.A. and Sokoloff, D.D. (1983) Magnetic Fields in Astrophysics, Gordon and Breach, New York, London, Paris.

KULSRUD: Is the root mean square field arising from dynamo action much larger than the mean field? If it is, the back reaction of the field becomes important before the mean field becomes appreciable. Thus, the dynamo theory should show $(\Delta B)^{2} \ll \bar{B}^{2}$ to be effective.

RUZMAIKIN: Yes, it can be larger. The distribution of the fluctuative field is typically intermittent, i.e. it concentrates into strong ropes or layers with a large space between them. After averaging one obtains a low mean field. To support this mean field a mean helicity is needed. 